\title{
A bibliometric analysis in gene research of myocardial infarction from 2001 to 2015
}

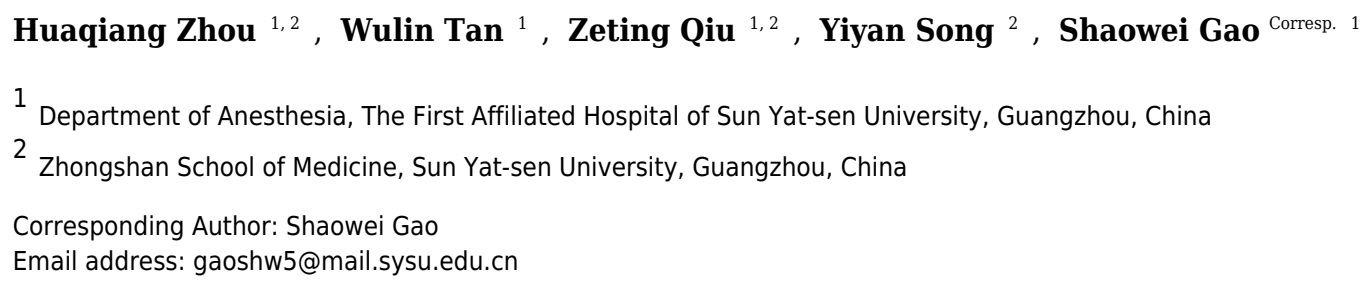

Objectives: We aimed to evaluate the global scientific output of gene research of myocardial infarction and explore their hotspots and frontiers from 2001 to 2015, using bibliometric methods. Methods: Articles about the gene research of myocardial infarction between 2001 and 2015 were retrieved from the Web of Science Core Collection (WoSCC). We used the bibliometric method and Citespace $V$ to analyze publication years, journals, countries, institutions, research areas, authors, research hotspots, and trends. We plotted the reference co-citation network, and we used key words to analyze the research hotspots and trends. Results: We identified 1853 publications on gene research of myocardial research from 2001 to 2015, and the annual publication number increased with time. Circulation published the highest number of articles. United States ranked highest in the countries with most publications, and the leading institute was Harvard University. Relevant publications were mainly in the field of Cardiovascular system cardiology. Keywords and references analysis indicated that gene expression, microRNA and young women were the research hotspots, whereas stem cell, chemokine, inflammation and cardiac repair were the frontiers. Conclusions: We depicted gene research of myocardial infarction overall by bibliometric analysis. Mesenchymal stem cells Therapy, MSCs-derived microRNA and genetic modified MSCs are the latest research frontiers. Related studies may pioneer the future direction of this filed in next few years. Further studies are needed. 
2

Huaqiang Zhou, MD a,b,\#, Wulin Tan, MD ${ }^{a, \#, ~ Z e t i n g ~ Q i u, ~ M D ~}{ }^{a, b, \#, ~ Y i y a n ~ S o n g, ~ M D ~}{ }^{b}$, Shaowei Gao, MD ${ }^{a, *}$

a Department of Anesthesia, The First Affiliated Hospital of Sun Yat-sen University,

b Zhongshan School of Medicine, Sun Yat-sen University, Guangzhou, China

\# Zhou, Tan and Qiu contributed equally to this work and should be regarded as co-first authors.

Correspondence information:

Department of Anesthesia, The First Affiliated Hospital of Sun Yat-sen University, 58

Zhongshan 2 Road, Guangzhou, China, 510080.

E-mail address: gaoshw5@mail.sysu.edu.cn Tel: +86 15013228745

Running head: Bibliometric Analysis about MI Genetics Research 


\section{Abstract}

Background: We aimed to evaluate the global scientific output of gene research of myocardial infarction and explore their hotspots and frontiers from 2001 to 2015, using bibliometric methods.

Methods: Articles about the gene research of myocardial infarction between 2001 and 2015 were retrieved from the Web of Science Core Collection (WoSCC). We used the bibliometric method and Citespace V to analyze publication years, journals, countries, institutions, research areas, authors, research hotspots, and trends. We plotted the reference co-citation network, and we used key words to analyze the research hotspots and trends.

Results: We identified 1853 publications on gene research of myocardial research from 2001 to 2015, and the annual publication number increased with time. Circulation published the highest number of articles. United States ranked highest in the countries with most publications, and the leading institute was Harvard University. Relevant publications were mainly in the field of Cardiovascular system cardiology. Keywords and references analysis indicated that gene expression, microRNA and young women were the research hotspots, whereas stem cell, chemokine, inflammation and cardiac repair were the frontiers.

Conclusions: We depicted gene research of myocardial infarction overall by bibliometric analysis. Mesenchymal stem cells Therapy, MSCs-derived microRNA and genetic modified MSCs are the latest research frontiers. Related studies may pioneer the future direction of this filed in next few years. Further studies are needed. 
45

\section{Introduction}

Myocardial infarction (MI), a life-threatening condition, occurs when lack of blood flow, causing the irreversible death of heart muscle. The incidence of MI has significantly increased in the past few decades, and MI remains a leading cause of death worldwide. With the development of genetic technology, gene researches have widely launched in MI. Researchers explore the genetics about MI and apply gene therapy for better treatment efficacy. Many genes have been identified an association with MI, such as PCSK9, TCF21(Braitsch et al. 2013; Kathiresan \& Myocardial Infarction Genetics 2008). Genome-wide association studies also have found 27 genetic variants that are associated with an increasing risk of MI(O'Donnell \& Nabel 2011). Although numerous papers focused on gene research of MI, there are limited attempts to analyze them systematically.

There are several bibliometric studies focused on cardiovascular diseases research. Mark et al performed a global bibliometric analysis from 1999 to 2008 to evaluate trends disaggregated by country(Huffman et al. 2013). Publications has increased substantially in the past decade, but the low-income countries with higher disease burdens still had a lower output. Bloomfield and AIKindi confirmed this phenomenon separately by the bibliometric analysis from 52 African countries and the Middle East(Al-Kindi et al. 2015; Bloomfield et al. 2015). Shuaib and et al. focused on the citation frequency of top 100 cited cardiovascular articles(Shuaib et al. 2015). However, a global bibliometric analysis of the gene research of MI has not yet been performed.

Bibliometric analysis is a widely used quantitative method to examine the knowledge structure and development in research fields(Guler et al. 2016). CiteSpace V is one of bibliometric 
66 visualization tools for visualizing and analyzing emerging trends and transition patterns in

67 scientific literature, which is developed by Chaomei Chen in 2004(Chen 2004; Chen 2006; Chen

68 et al. 2010; Synnestvedt et al. 2005). Now it has been widely used to evaluate the productivity of

69 institutions, countries and authors; identify international collaborations and geographic

70 distributions; and explore research hotspots and frontiers in specified fields(Chen et al. 2014). In

71 the present study, we performed a bibliometric analysis of articles on the gene research of MI

72 indexed from 2001 to 2015, by using CiteSpace V to explore the research trend and hot spots.

\section{Materials \& Methods}

We performed online retrieval from the Web of Science Core Collection (WoSCC) of Thomson Reuters on Oct 1, 2016. We used the key words "myocardial infarction" and "gene" to retrieve research articles or reviews between 2001 and 2015. We collected the following basic info for each article: authors, title, abstract, institution, country/region, journal, keywords, and references. The search queries were listed below in Supplementary Table 1.

Articles or reviews that meet the following criteria were included: (1) The time span is between 2001 and 2015. (2) Articles indexed in WoSCC. (3) Articles on gene research of MI, including original research and reviews. (4) Articles with basic info. The following document were excluded: (1) Meeting abstracts, proceedings, corrected articles, and repeated articles. (2) Unpublished Documents without enough information for further analysis.

The downloaded data were analyzed based on Web of Science database literature analysis report and export information function. Then we used CiteSpace V (64 bits) to analyze publication outputs and construct knowledge maps. In addition, we also used software VOSviewer (version 
88

89

90

91

92

93

94

95

96

1.6.6) for better network visualizations in some cases(Ozsoy \& Demir 2017; van Eck \& Waltman 2010). In this paper, Individual network was derived from the 50 most cited articles published in a one-year time slice. TFIDF weighting was used to analyze the content of each cluster(Merigo \& Montserrat 2010). Finally, we also applied burst detection to investigate the growth rate of citations or keywords(Lee et al. 2016).

\section{Results}

Based on the selection criteria, 1,853 publications about gene research of MI were indexed in WOSCC from 2001 to 2015, and were included in the study. Much of publications were research articles $(1799,97.1 \%)$, followed by review articles $(54,2.9 \%)$. English $(98.4 \%)$ is primary language used by scholars, while the remaining $1.6 \%$ of the publications were written in Russian, Spanish, German and Portuguese. The number of publications (Supplementary Table 2) showed a gradually increased trend over time which rose from 82 in 2001 to 164 in 2015 and peaked in 2015.

More than 494 scholarly journals have published articles on MI research. The 10 most active journals are presented in Supplementary Table 3. They published 444 articles and accounted for $24.0 \%$ of all articles included in this study. Circulation published the highest number of articles (66, 3.616\%), followed by Atherosclerosis (60, 3.238\%), Plos One (48, 2.590\%), American Journal of Physiology Heart and Circulatory Physiology and Journal of Molecular (42, 2.267\%) and Cellular Cardiology (42, 2.267\%).

The 1,853 articles originated from 69 countries and territories (Table 1, Figure 1). The top 10 countries are composed of six European countries, two Asian countries and two North American 
109 countries which accounted for $94.87 \%$ of the total number of publications. USA, China, Japan,

110 Germany and Italy were the most productive countries.

111 The 1,853 articles were published by more than 500 research institutions (Table 1, Figure 2).

112 The top 10 institutions with the greatest outputs in this area totally published 351 articles, 113 accounting for $18.94 \%$ of publications. The first major research echelon was led by Harvard 114 University, followed by Karolinska Institutet, University of Washington, University of Toronto, 115 and Leiden University.

116 Gene research of MI were distributed in more than 60 special research areas. Supplementary 117 Table 4 shows the top 10 research areas from 2001 to 2015. Cardiovascular System Cardiology 118 accounted for the largest number of publications $(45.278 \%)$, followed by research experimental 119 medicine $(11.387 \%)$, genetics heredity $(10.362 \%)$, cell biology $(9.498 \%)$, and hematology $120(8.852 \%)$.

The 1853 articles were written by more than 11225 authors. The top 10 authors publishing 122 articles were listed in Supplementary Table 5. Hamsten Anders from Karolinska Institutet, Psaty $\mathrm{BM}$ and Schunkert $\mathrm{H}$ published the most articles (25 records) and accounted for $1.349 \%$ of all 124 published articles. We also analyzed author citations, by using Citespace V, and constructed co125 citation maps to evaluate the scientific relevance of publications. As shown in Figure 3, the largest nodes were Pfeffer MA (179 citations), Ridker PM (143 citations), Frangogiannis NG (125 citations) and Libby P (119 citations), indicating their important role in MI research. Additionally, 128 there were 7 large citation clusters in Figure 3.

129 In the present study, we used CiteSpace V to construct a knowledge map of keyword co130 occurrence with 147 nodes and 947 links (Figure 4) and identified the top 20 keywords in 
131 publications from 2001 to 2015 (Supplementary Table 6), according to frequency and citation

132 counts. The top keywords were 'myocardial infarction', 'coronary artery disease', 'heart failure',

133 'expression', 'polymorphism', 'atherosclerosis', and 'gene expression'.

134 We used CiteSpace V to detect burst keywords. Burst keywords are considered as indicators of 135 research frontiers or emerging trends over time. Figure 5 shows the top 20 keywords with the 136 strongest citation bursts. The strongest ones include cytokine, angiotensin converting enzyme, 137 genetics, mutation and mesenchymal stem cell. The recent burst of keywords were microRNA, 138 mesenchymal stem cell, oxidative stress and gene therapy.

139 We constructed a co-cited reference map and explored changes associated with the key clusters 140 of articles. The network contains 491 nodes and 1374 links. The Modularity Q was 0.7863 and the 141 Mean Silhouette was 0.3218 (Figure 6). There were initially 71 clusters, and we filtered out small 142 clusters with low silhouette. To learn more about the development of a cluster in a certain period, 143 we also construct a reference co-citation time-view map (Figure 7). According to the citation 144 frequency and centricity, we selected hotspot references in each period of time.

\section{Discussion}

147 When considered with the impact of a journal, we found that the top 10 active journals all had 148 an IF $>3.0$, and more than $33.3 \%(3 / 10)$ of the top 10 active journals had an IF $>10$ which account 149 for $7 \%$ of the total number of included publications, including Circulation (IF2015=17.047),

150 Journal of the American College of Cardiology (IF2015=17.759) and Circulation Research 151 (IF2015=11.551). When compared with the high-IF rate of all MI articles $(7.5 \%)$, gene research 152 of MI was still intensively published in high-IF journals. 
153

154

155

156

157

158

159

160

161

162

163

164

165

166

167

168

169

170

171

172

173

174

175

The United States was the leading country in gene research of MI over the past 15 years, and China was the only developing country in the leading group, demonstrating its significant progress in the life sciences during the past 15 years. Our study is not the first investigation showing the pioneer countries in scientific output. These results were roughly consistent with previous cardiovascular disease bibliometric studies(Huffman et al. 2013). A 2017 study showed that the USA has the highest number of strong citation links with other countries, and China has highest growth(Gal et al. 2017). Although the number of publications from Latin America, Africa, and the Middle East increased in the past decade, these low- and middle-income countries with higher disease burdens still lag behinds developed countries, which are needed more target research investments and international collaboration(Al-Kindi et al. 2015; Bloomfield et al. 2015; Colantonio et al. 2015; Jahangir et al. 2011).

In Figure 3, the color of nodes indicated how new the associated reference was. Cool colors referred to old researches, warm colors new researches(Chen et al. 2012). Consequently, Ridker PM (in the bottom) and Pfeffer MA (in the top) represents the basis of MI research. The former concentrates on clinical associations, such as hyperhomocysteinemia, atherosclerosis and so on(Libby et al. 2011; Zee et al. 2007). The latter hammers focus on the pathophysiology, such as changes of the renal function(Verma et al. 2007). In the middle of Figure 3, warm-color nodes and linkages, leaded by Frangogiannis NG and Libby $\mathrm{P}$, indicated that transcriptome was new directions(Fiedler et al. 2011; Ounzain et al. 2015). Young women label appeared twice in warmcolor clusters, contacting closely with genotype, which may have indicated young MI women may be associated with genetics variants. Some articles found that young women with acute myocardial infarction have more comorbidity and higher in-hospital mortality than young men(Gupta et al. 2014). Young women with MI more often have a family history of premature MI, which was 
176 addressed in a study of 398 families in which 62 vascular biology genes were evaluated. Single

177 nucleotide polymorphisms in several thrombospondin genes were significantly associated with

178 familial premature MI(Topol et al. 2001).

179 The topics involved in gene research of MI can been delineated in the keywords assigned to

180

181

182

183

184

185

186

187

each article. Keywords provide a reasonable description of research hotspots, whereas burst words represent new research frontiers(Chen et al. 2014). Adjacent keywords are often assigned to the same articles. For example, in Supplementary Table 6, 'coronary artery disease', 'atherosclerosis', 'myocardial infarction', and 'heart failure' are closely related to coronary artery disease, and are different pathophysiology stages of CHD. Gene expression is the synthesis process of gene product which leads to the appearance in the phenotype. There are many gene products being associated with MI, such as microRNA, SDF1 and so on(Boon \& Dimmeler 2015; Bromage et al. 2014). Burst words can be detected by CiteSpace, which represent words that are cited frequently in a period of time. As shown in Figure 5, the top 4 burst keywords were as follow, mesenchymal stem cell, oxidative stress, injury, and microRNA. Combined with those keywords with red lines in Figure 4 for further analysis, mesenchymal stem cells (MSCs) transplantation, microRNA and cardiac repair are forefronts of research in recent years. MSCs is a kind of non-hematopoietic stem cell with low immunogenicity, which has become the main cell for MI regenerative therapy(Chou et al. 2014). Many studies showed that MSCs transplantation therapy could have infarct-limiting and cardiac repair effects after MI by revitalizing the cardiac stem cell and revascularization(Russo et al. 2014; Shafei et al. 2017). Paracrine effect of MSCs could be mediated by extracellular vesicles, such as MSCs-derived exosomal microRNA(Huang et al. 2015; Wen et al. 2012). Genetic modified MSCs has been a therapeutic hotspot target for MI, which can promote secretion of paracrine factors to enhance the therapeutic effect(Karpov et al. 2017). 
As is shown in Figure 6 and Supplementary Table 7, Cluster 0, 1, 4, and 5 play an important role. Cluster 0 (basic-fibroblast growth factor | gene expression profiles) was the largest while Cluster 1 (system biology / cell survival) and Cluster 4 (inflammation / young women) have the most burst cited-articles. The nodes and linkages of Cluster 2 (Chemokine / atherogenesis) and Cluster 5 (cardiac repair) are painted on warm color, and indicated they are the latest research, which is better visualized in Figure 7. Besides the same warm colors, Cluster 2 and Cluster 5 have related overlapping each other, indicating relevance from aspect of literature metrology. Upregulation of chemokines is a hallmark of the inflammatory following MI(Cavalera \& Frangogiannis 2014). Some researchers have suggested that chemokines may be potential therapeutic targets to promote wholesome cardiac repair in MI patients(Cavalera \& Frangogiannis 2014). It just so happens that "young women" appear once again in the Cluster 4 echoing with the previous keywords analysis, hint a hotspot in the gene research of MI(Spatz et al. 2015).

There are some limitations in our bibliometric study. The primary source of input data for CiteSpace is the WoSCC, which is more advanced at getting detailed data (e.g. journal sources, author, country and institution information). So, our analysis was performed only with publications in the WoSCC without using multiple search engines (Pubmed, Ovid, Scopus And Google Scholar et al. are not included). In addition, a linguistic bias may exist because most publications in the WosCC were in English.

\section{Conclusions}

In summary, we depicted the scientific output of gene research of myocardial infarction by bibliometric analysis overall. Mesenchymal stem cells Therapy, MSCs-derived microRNA and 
221 genetic modified MSCs are the latest research frontiers. Related studies may pioneer the future

222 direction of this filed in next few years. Further studies are needed.

223

\section{Acknowledgement}

225 The authors would like to thank editors and the anonymous reviewers for their valuable 226 comments and suggestions to improve the quality of the paper. They are also grateful to CDMG 227 (SYSU) for assistance. 


\section{References}

Al-Kindi S, Al-Juhaishi T, Haddad F, Taheri S, and Abi Khalil C. 2015. Cardiovascular disease research activity in the Middle East: a bibliometric analysis. Ther Adv Cardiovasc Dis 9:70-76. 10.1177/17539447715578585

Bloomfield GS, Baldridge A, Agarwal A, Huffman MD, Colantonio LD, Bahiru E, Ajay VS, Prabhakaran P, Lewison G, and Prabhakaran D. 2015. Disparities in cardiovascular research output and citations from 52 African countries: a time-trend, bibliometric analysis (1999-2008). J Am Heart Assoc 4. 10.1161/JAHA.114.001606

Boon RA, and Dimmeler S. 2015. MicroRNAs in myocardial infarction. Nat Rev Cardiol 12:135-142. 10.1038/nrcardio.2014.207

Braitsch CM, Kanisicak O, van Berlo JH, Molkentin JD, and Yutzey KE. 2013. Differential expression of embryonic epicardial progenitor markers and localization of cardiac fibrosis in adult ischemic injury and hypertensive heart disease. J Mol Cell Cardiol 65:108-119. 10.1016/j.yjmcc.2013.10.005

Bromage DI, Davidson SM, and Yellon DM. 2014. Stromal derived factor 1 $\alpha$ : a chemokine that delivers a twopronged defence of the myocardium. Pharmacol Ther 143:305-315. 10.1016/j.pharmthera.2014.03.009

Cavalera M, and Frangogiannis NG. 2014. Targeting the chemokines in cardiac repair. Curr Pharm Des 20:19711979.

Chen C. 2004. Searching for intellectual turning points: progressive knowledge domain visualization. Proc Natl Acad Sci U S A 101 Suppl 1:5303-5310. 10.1073/pnas.0307513100

Chen C. 2006. CiteSpace II: Detecting and visualizing emerging trends and transient patterns in scientific literature. $J$ Am Soc Inf Sci 57:359-377. 10.1002/asi.20317

Chen C, Dubin R, and Kim MC. 2014. Emerging trends and new developments in regenerative medicine: a scientometric update (2000 - 2014). Expert Opin Biol Ther 14:1295-1317. 10.1517/14712598.2014.920813

Chen C, Hu Z, Liu S, and Tseng H. 2012. Emerging trends in regenerative medicine: a scientometric analysis in CiteSpace. Expert Opin Biol Ther 12:593-608. 10.1517/14712598.2012.674507

Chen C, Ibekwe-SanJuan F, and Hou J. 2010. The structure and dynamics of cocitation clusters: A multipleperspective cocitation analysis. J Am Soc Inf Sci 61:1386-1409. 10.1002/asi.21309

Chou S-H, Lin S-Z, Kuo W-W, Pai P, Lin J-Y, Lai C-H, Kuo C-H, Lin K-H, Tsai F-J, and Huang C-Y. 2014. Mesenchymal stem cell insights: prospects in cardiovascular therapy. Cell Transplant 23:513-529. $10.3727 / 096368914$ X678436

Colantonio LD, Baldridge AS, Huffman MD, Bloomfield GS, and Prabhakaran D. 2015. Cardiovascular research publications from Latin America between 1999 and 2008. A bibliometric study. Arq Bras Cardiol 104:5-15. $10.5935 / \mathrm{abc} .20140213$

Fiedler J, Jazbutyte V, Kirchmaier BC, Gupta SK, Lorenzen J, Hartmann D, Galuppo P, Kneitz S, Pena JTG, SohnLee C, Loyer X, Soutschek J, Brand T, Tuschl T, Heineke J, Martin U, Schulte-Merker S, Ertl G, Engelhardt S, Bauersachs J, and Thum T. 2011. MicroRNA-24 regulates vascularity after myocardial infarction. Circulation 124:720-730. 10.1161/CIRCULATIONAHA.111.039008

Gal D, Glänzel W, and Sipido KR. 2017. Mapping cross-border collaboration and communication in cardiovascular research from 1992 to 2012. Eur Heart J 38:1249-1258. 10.1093/eurheartj/ehw459

Guler AT, Waaijer CJF, and Palmblad M. 2016. Scientific workflows for bibliometrics. Scientometrics 107:385-398. $10.1007 / \mathrm{s} 11192-016-1885-6$

Gupta A, Wang Y, Spertus JA, Geda M, Lorenze N, Nkonde-Price C, D'Onofrio G, Lichtman JH, and Krumholz HM. 2014. Trends in acute myocardial infarction in young patients and differences by sex and race, 2001 to 2010. J Am Coll Cardiol 64:337-345. 10.1016/j.jacc.2014.04.054

Huang L, Ma W, Ma Y, Feng D, Chen H, and Cai B. 2015. Exosomes in mesenchymal stem cells, a new therapeutic 
284

285

286

287

288

289

290

291

292

293

294

295

296

297

298

299

300

301

302

303

304

305

306

307

308

309

310

311

312

313

314

315

316

317

318

319

320

321

322

323

324

325

326

327

328

329

330

331

332

333

334

335

336

337

338

339

strategy for cardiovascular diseases? Int J Biol Sci 11:238-245. 10.7150/ijbs.10725

Huffman MD, Baldridge A, Bloomfield GS, Colantonio LD, Prabhakaran P, Ajay VS, Suh S, Lewison G, and Prabhakaran D. 2013. Global cardiovascular research output, citations, and collaborations: a time-trend, bibliometric analysis (1999-2008). PLoS One 8:e83440. 10.1371/journal.pone.0083440

Jahangir E, Comandé D, and Rubinstein A. 2011. Cardiovascular disease research in Latin America: a comparative bibliometric analysis. World J Cardiol 3:383-387. 10.4330/wjc.v3.i12.383

Karpov AA, Udalova DV, Pliss MG, and Galagudza MM. 2017. Can the outcomes of mesenchymal stem cell-based therapy for myocardial infarction be improved? Providing weapons and armour to cells. Cell Prolif 50. 10.1111/cpr.12316

Kathiresan S, and Myocardial Infarction Genetics C. 2008. A PCSK9 missense variant associated with a reduced risk of early-onset myocardial infarction. N Engl J Med 358:2299-2300. 10.1056/NEJMc0707445

Lee Y-C, Chen C, and Tsai X-T. 2016. Visualizing the Knowledge Domain of Nanoparticle Drug Delivery Technologies: A Scientometric Review. NATO Adv Sci Inst Ser E Appl Sci 6:11. 10.3390/app6010011

Libby P, Ridker PM, and Hansson GK. 2011. Progress and challenges in translating the biology of atherosclerosis. Nature 473:317-325. 10.1038/nature10146

Merigo JM, and Montserrat C. 2010. Induced and heavy aggregation operators with distance measures. Journal of Systems Engineering and Electronics 21:431-439. 10.3969/j.issn.1004-4132.2010.03.013

O'Donnell CJ, and Nabel EG. 2011. Genomics of cardiovascular disease. N Engl J Med 365:2098-2109. 10.1056/NEJMra1105239

Ounzain S, Micheletti R, Beckmann T, Schroen B, Alexanian M, Pezzuto I, Crippa S, Nemir M, Sarre A, Johnson R, Dauvillier J, Burdet F, Ibberson M, Guigó R, Xenarios I, Heymans S, and Pedrazzini T. 2015. Genome-wide profiling of the cardiac transcriptome after myocardial infarction identifies novel heart-specific long noncoding RNAs. Eur Heart J 36:353-368a. 10.1093/eurheartj/ehu180

Ozsoy Z, and Demir E. 2017. The Evolution of Bariatric Surgery Publications and Global Productivity: A Bibliometric Analysis. Obes Surg. 10.1007/s11695-017-2982-1

Russo V, Young S, Hamilton A, Amsden BG, and Flynn LE. 2014. Mesenchymal stem cell delivery strategies to promote cardiac regeneration following ischemic injury. Biomaterials 35:3956-3974. 10.1016/j.biomaterials.2014.01.075

Shafei AE-S, Ali MA, Ghanem HG, Shehata AI, Abdelgawad AA, Handal HR, Talaat KA, Ashaal AE, and El-Shal AS. 2017. Mesenchymal stem cell therapy: A promising cell-based therapy for treatment of myocardial infarction. J Gene Med 19. 10.1002/jgm.2995

Shuaib W, Khan MS, Shahid H, Valdes EA, and Alweis R. 2015. Bibliometric analysis of the top 100 cited cardiovascular articles. Am J Cardiol 115:972-981. 10.1016/j.amjcard.2015.01.029

Spatz ES, Curry LA, Masoudi FA, Zhou S, Strait KM, Gross CP, Curtis JP, Lansky AJ, Soares Barreto-Filho JA, Lampropulos JF, Bueno H, Chaudhry SI, D'Onofrio G, Safdar B, Dreyer RP, Murugiah K, Spertus JA, and Krumholz HM. 2015. The Variation in Recovery: Role of Gender on Outcomes of Young AMI Patients (VIRGO) Classification System: A Taxonomy for Young Women With Acute Myocardial Infarction. Circulation 132:1710-1718. 10.1161/CIRCULATIONAHA.115.016502

Synnestvedt MB, Chen C, and Holmes JH. 2005. CiteSpace II: visualization and knowledge discovery in bibliographic databases. AMIA Annu Symp Proc:724-728.

Topol EJ, McCarthy J, Gabriel S, Moliterno DJ, Rogers WJ, Newby LK, Freedman M, Metivier J, Cannata R, O'Donnell CJ, Kottke-Marchant K, Murugesan G, Plow EF, Stenina O, and Daley GQ. 2001. Single nucleotide polymorphisms in multiple novel thrombospondin genes may be associated with familial premature myocardial infarction. Circulation 104:2641-2644.

van Eck NJ, and Waltman L. 2010. Software survey: VOSviewer, a computer program for bibliometric mapping. Scientometrics 84:523-538. 10.1007/s11192-009-0146-3

Verma A, Anavekar NS, Meris A, Thune JJ, Arnold JMO, Ghali JK, Velazquez EJ, McMurray JJV, Pfeffer MA, and Solomon SD. 2007. The relationship between renal function and cardiac structure, function, and prognosis after myocardial infarction: the VALIANT Echo Study. $J$ Am Coll Cardiol 50:1238-1245. 10.1016/j.jacc.2007.06.018

Wen Z, Zheng S, Zhou C, Yuan W, and others. 2012. Bone marrow mesenchymal stem cells for post-myocardial infarction cardiac repair: micrornas as novel regulators. Journal of cellular.

Zee RYL, Mora S, Cheng S, Erlich HA, Lindpaintner K, Rifai N, Buring JE, and Ridker PM. 2007. Homocysteine, 5,10-methylenetetrahydrofolate reductase $677 \mathrm{C}>\mathrm{T}$ polymorphism, nutrient intake, and incident cardiovascular disease in 24,968 initially healthy women. Clin Chem 53:845-851. 10.1373/clinchem.2006.083881 
Figure 1

Map of countries and territories of groups that published articles on the gene research of myocardial infarction during 2001-2015.

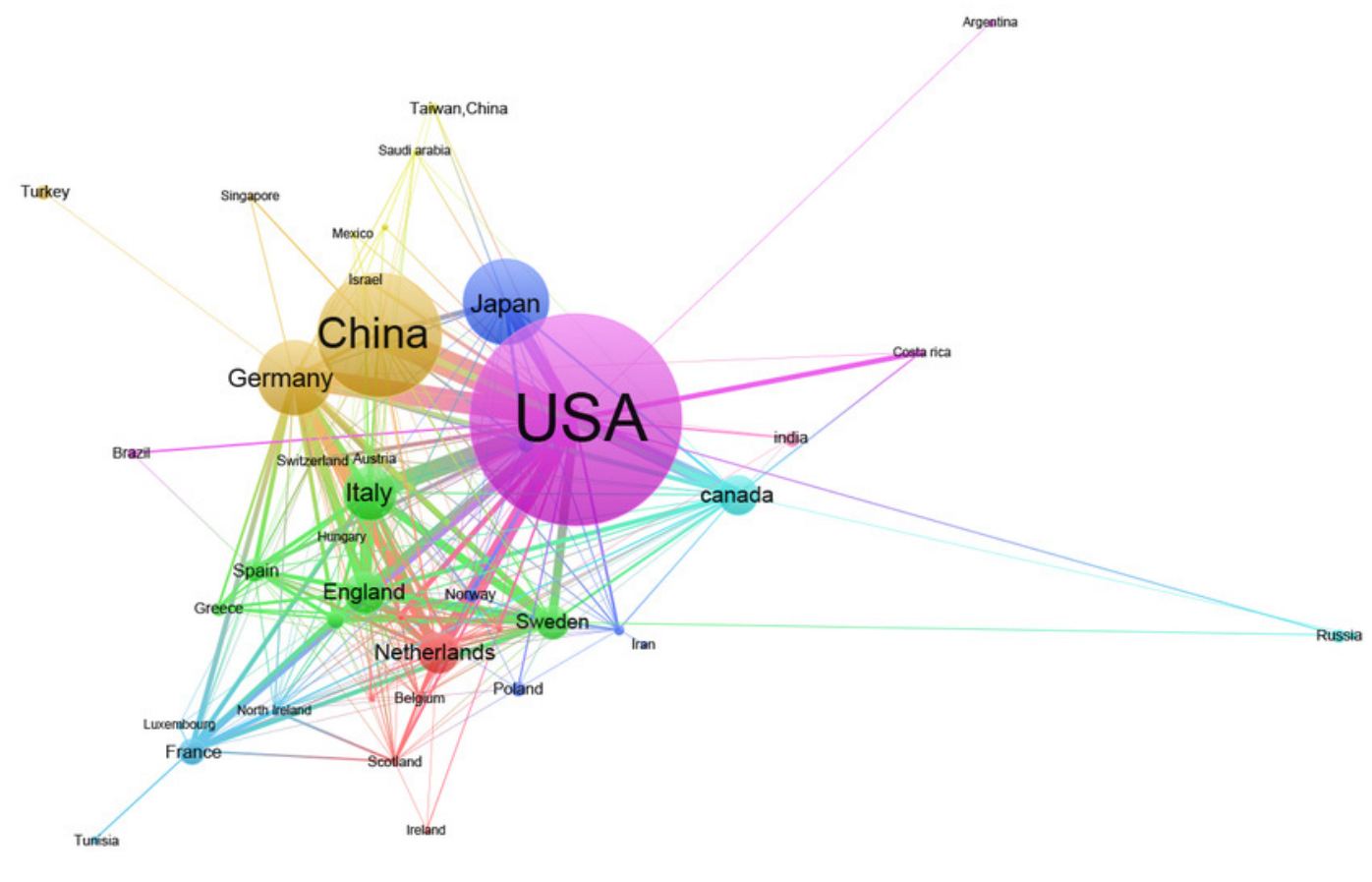




\section{Figure 2}

Institutions that published articles on the gene research of myocardial infarction during 2001-2015.

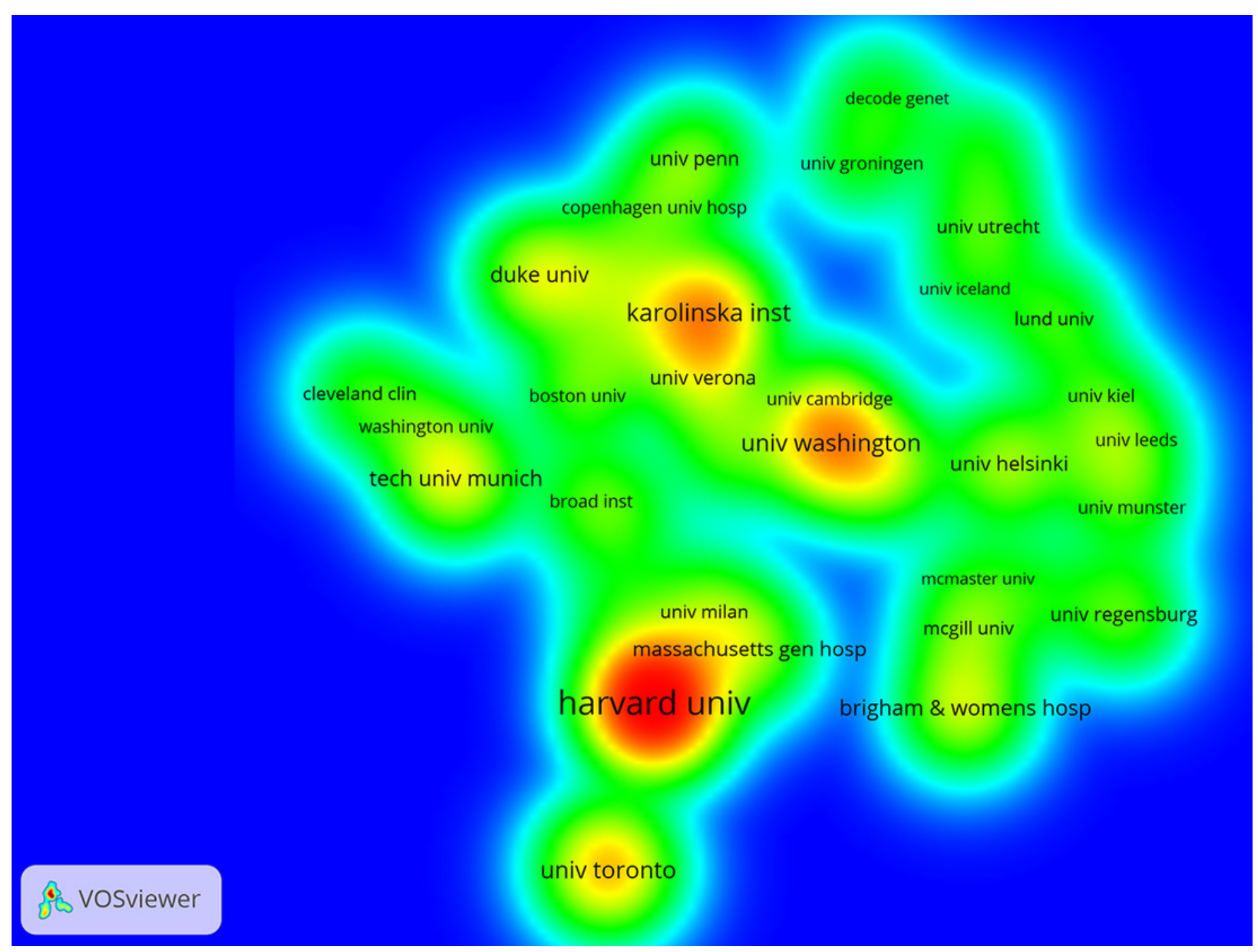


Figure 3

Co-citation map of authors who published articles on the gene research of myocardial infarction during 2001-2015

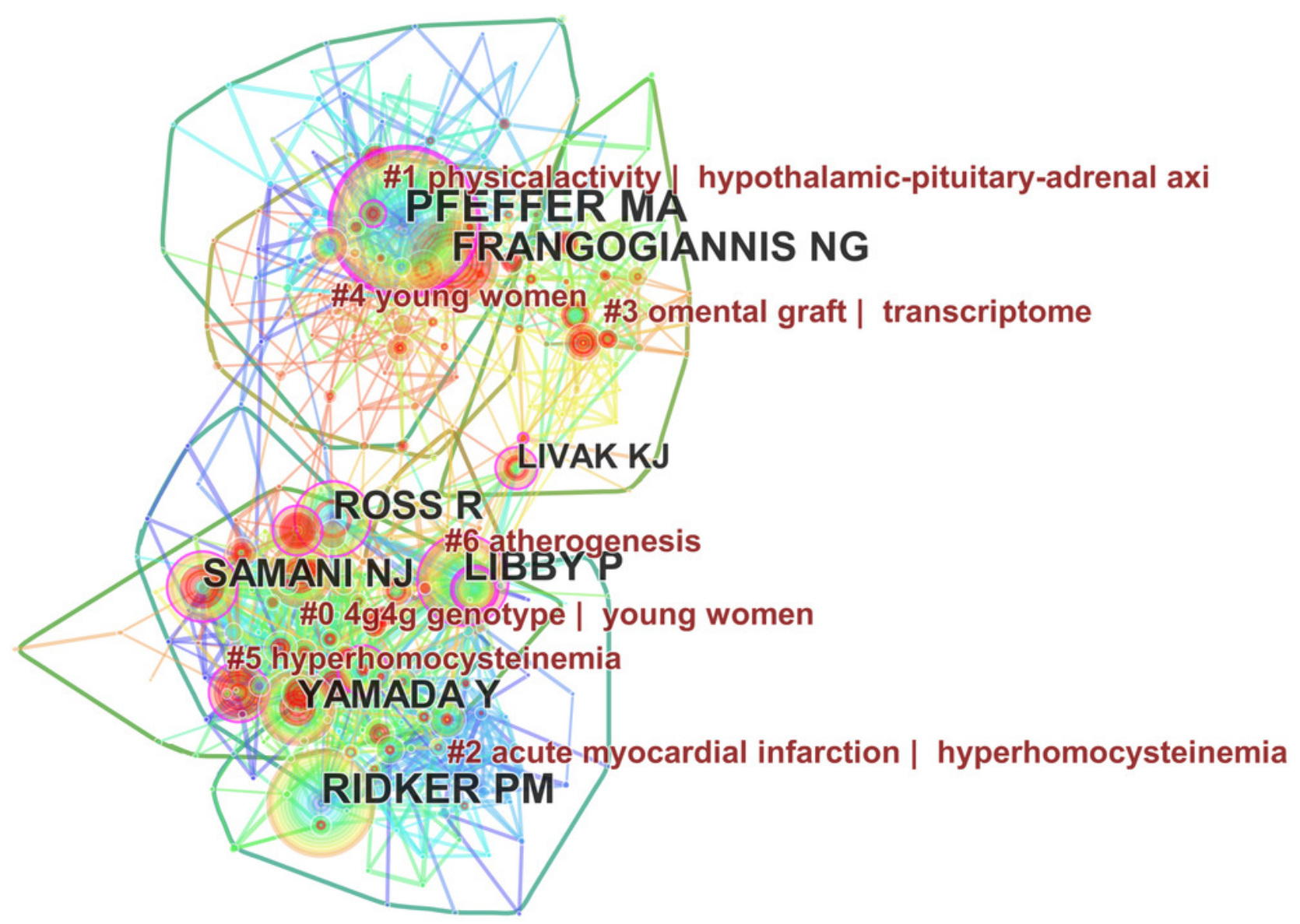




\section{Figure 4}

Keywords networks based on articles on the gene research of myocardial infarction during 2001-2015

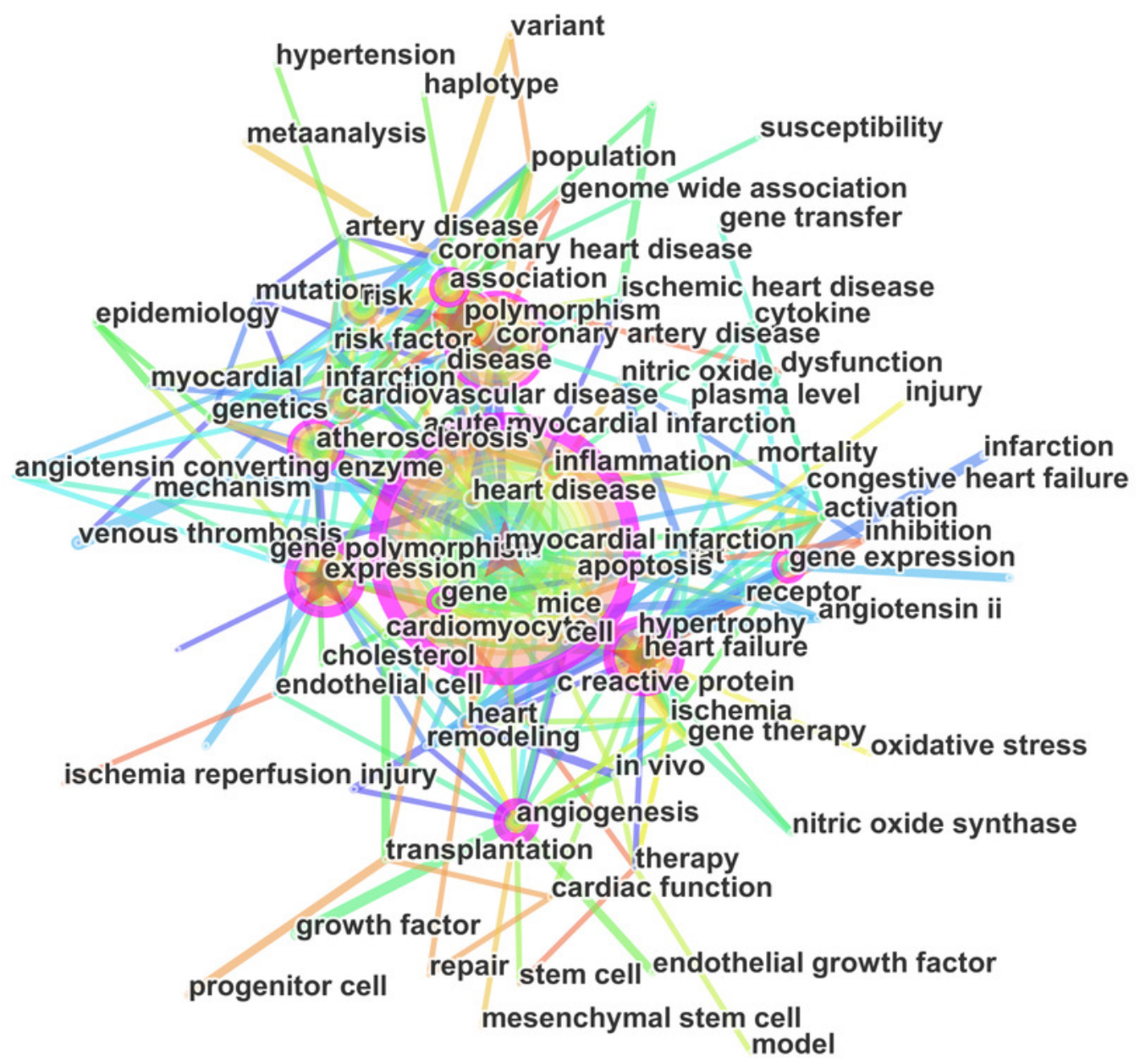




\section{Figure 5}

Top 20 keywords with the strongest citation bursts on the gene research of myocardial infarction during 2001-2015

\section{Top 20 Keywords with the Strongest Citation Bursts}

\begin{tabular}{|c|c|c|c|c|c|}
\hline Keywords & Year & Strength & Begin & End & 2001-2015 \\
\hline cytokine & 2001 & 13.4142 & 2002 & 2006 & 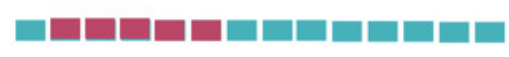 \\
\hline angiotensin converting enzyme & 2001 & 13.0615 & 2001 & 2006 & 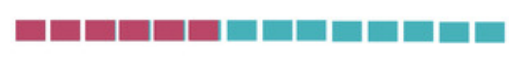 \\
\hline genetics & 2001 & 11.665 & 2006 & 2009 & 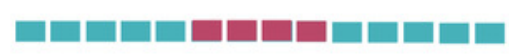 \\
\hline mutation & 2001 & 10.4072 & 2001 & 2004 & 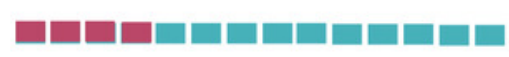 \\
\hline mesenchymal stem cell & 2001 & 9.8746 & 2011 & 2015 & 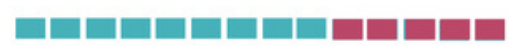 \\
\hline injury & 2001 & 9.8208 & 2012 & 2015 & 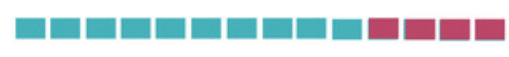 \\
\hline endothelial growth factor & 2001 & 9.7786 & 2008 & 2010 & 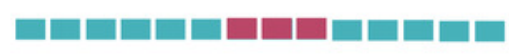 \\
\hline oxidative stress & 2001 & 9.5117 & 2012 & 2015 & 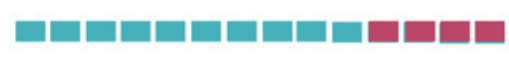 \\
\hline microrna & 2001 & 9.1851 & 2013 & 2015 & 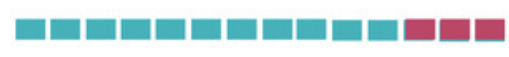 \\
\hline haplotype & 2001 & 9.0539 & 2006 & 2009 & 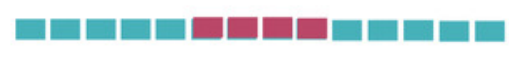 \\
\hline congestive heart failure & 2001 & 9.0239 & 2003 & 2005 & 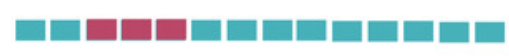 \\
\hline stem cell & 2001 & 8.908 & 2010 & 2012 & 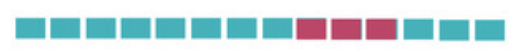 \\
\hline cardiac function & 2001 & 8.888 & 2010 & 2015 & 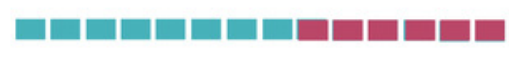 \\
\hline rat & 2001 & 8.3051 & 2001 & 2005 & 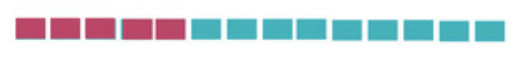 \\
\hline infarction & 2001 & 8.1902 & 2001 & 2005 & 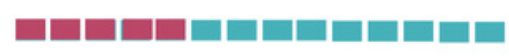 \\
\hline remodeling & 2001 & 8.1658 & 2005 & 2007 & 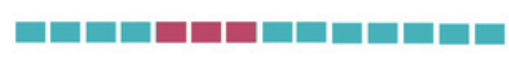 \\
\hline gene therapy & 2001 & 7.9672 & 2011 & 2013 & 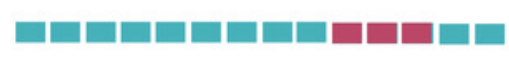 \\
\hline nitric oxide synthase & 2001 & 7.6665 & 2006 & 2007 & 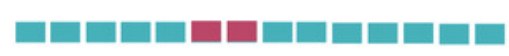 \\
\hline gene & 2001 & 7.5736 & 2001 & 2003 & 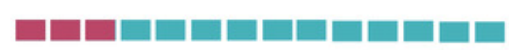 \\
\hline angiotensin ii & 2001 & 7.4706 & 2003 & 2005 & 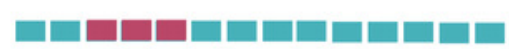 \\
\hline
\end{tabular}




\section{Figure 6}

Reference co-citation map of articles on the gene research of myocardial infarction during 2001-2015

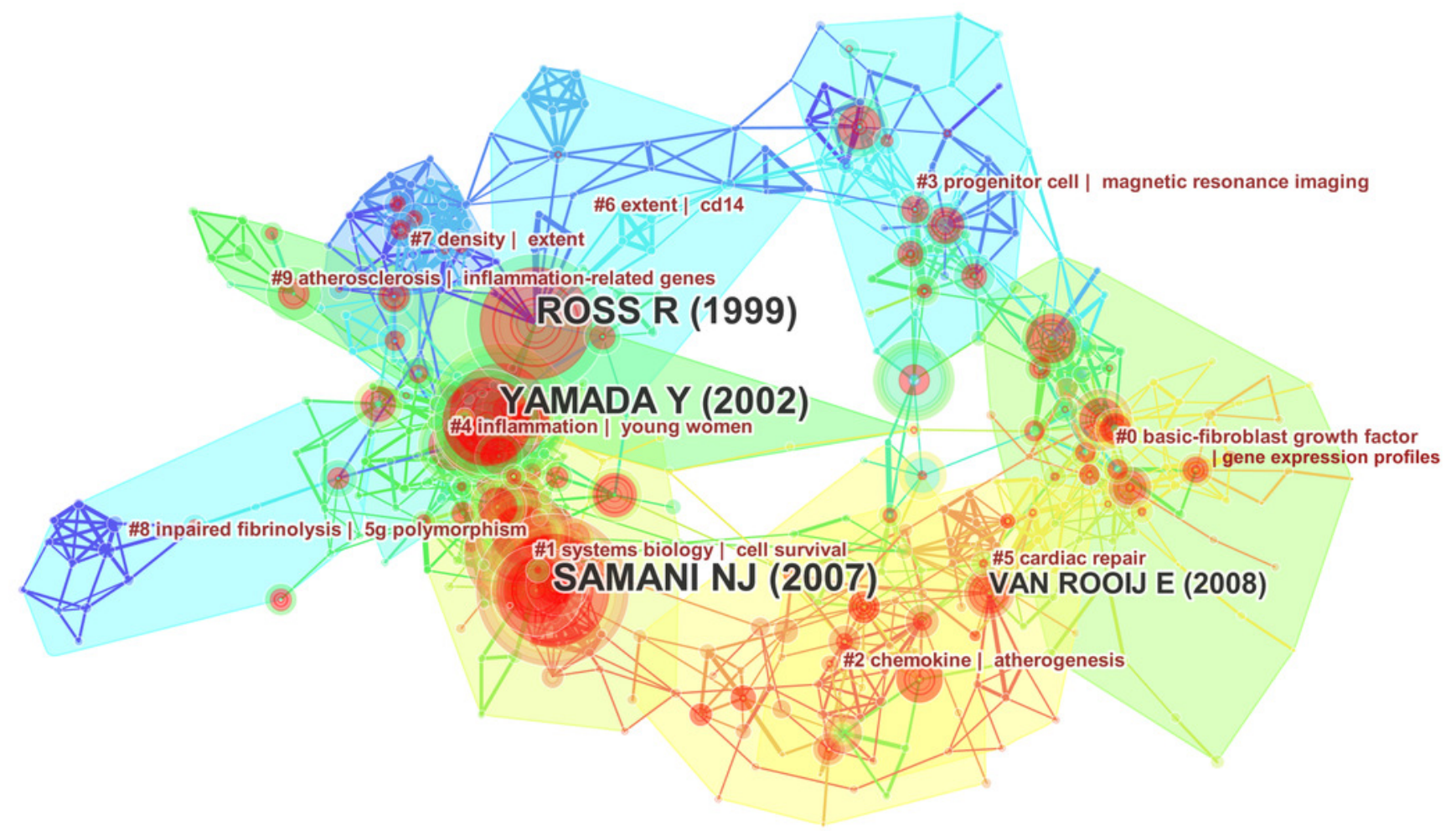


Figure 7

Reference co-citation time-view map of articles on the gene research of myocardial infarction during 2001-2015

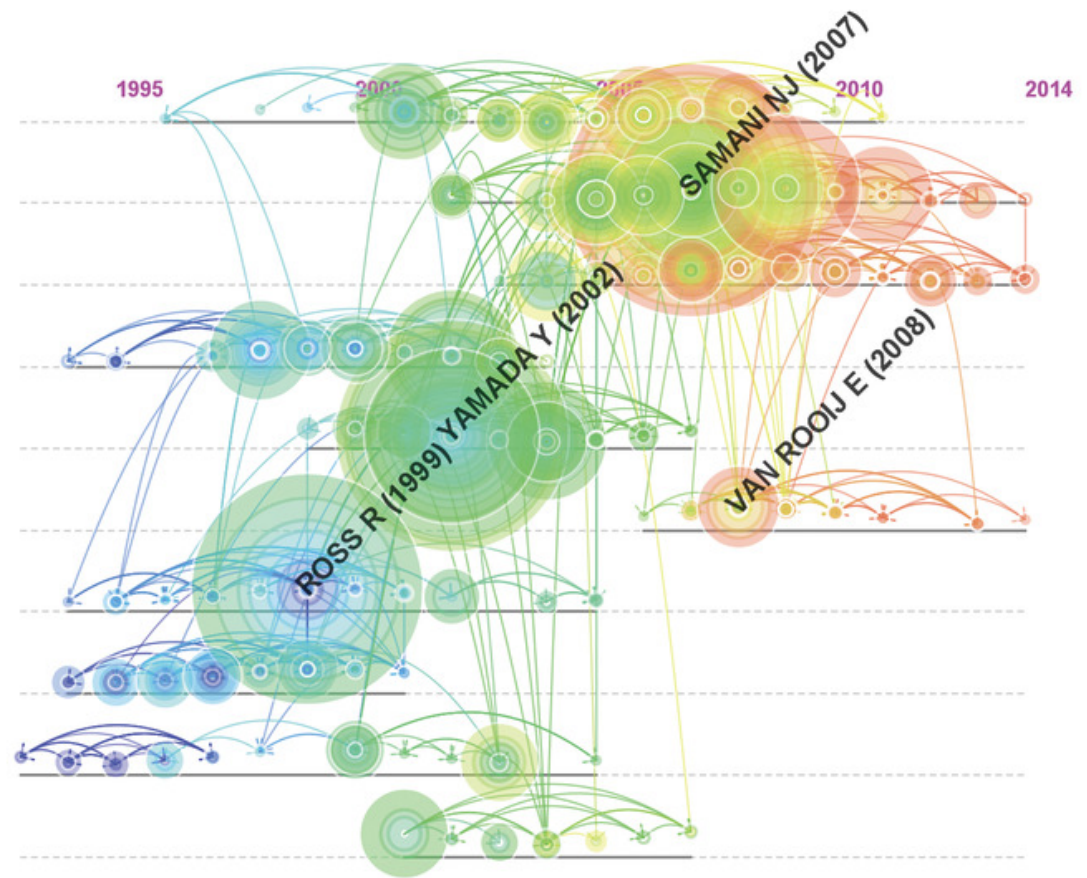

\#0 basic-fibroblast growth factor | gene expression profiles

\#1 systems biology | cell survival

\#2 chemokine | atherogenesis

\#3 progenitor cell | magnetic resonance imaging

\#4 inflammation | young women

\#5 cardiac repair

\#6 extent | cd14

\#7 density | extent

\#8 inpaired fibrinolysis | $5 \mathrm{~g}$ polymorphism

\#9 atherosclerosis | inflammation-related genes 


\section{Table $\mathbf{1}$ (on next page)}

Ranking of countries and institutions that published articles on the gene research of myocardial infarction indexed in the Web of Science during 2001-2015 
1 Table 1 Ranking of countries and institutions that published articles on the gene research of 2 myocardial infarction indexed in the Web of Science during 2001-2015

\begin{tabular}{clrlr}
\hline \multirow{2}{*}{ Rank } & Country & Counts(\%) & Institution & Counts(\%) \\
\hline 1 & USA & $509(27.469)$ & HARVARD UNIV & $88(4.749)$ \\
2 & CHINA & $297(16.028)$ & KAROLINSKA INST & $37(1.997)$ \\
3 & JAPAN & $205(11.063)$ & UNIV WASHINGTON & $35(1.889)$ \\
4 & GERMANY & $180(9.714)$ & UNIV TORONTO \\
5 & ITALY & $128(6.908)$ & LEIDEN UNIV & $35(1.889)$ \\
6 & ENGLAND & $102(5.505)$ & HUAZHONG UNIV SCI TECHNOL & $29(1.565)$ \\
7 & NETHERLAND & $97(5.235)$ & KAROLINSKA UNIV HOSP & $28(1.511)$ \\
8 & CANADA & $97(5.235)$ & OSAKA UNIV & $27(1.457)$ \\
9 & SWEDEN & $81(4.371)$ & TECH UNIV MUNICH & $25(1.349)$ \\
10 & FRANCE & $62(3.346)$ & DUKE UNIV & $24(1.295)$ \\
\hline
\end{tabular}

3 